\title{
Ambulatório de psiquiatria infantil: prevalência de transtornos mentais em crianças e adolescentes
}

Claudemir Marcos Machado

Bolsista de Iniciação Científica da Faculdade de Medicina de São José do Rio Preto, São José do Rio Preto - SP - Brasil

Andreia Mara Angelo Gonçalves Luiz Serviço de Psicologia do Hospital de Base, São José do Rio Preto - SP - Brasil

Altino Bessa Marques Filho
Maria Cristina de Oliveira Santos Miyazaki'
Neide Aparecida Micelli Domingos
Eliana Márcia Sotello Cabrera Faculdade de Medicina de São José do Rio Preto - SP - Brasil

\begin{abstract}
Resumo: Este estudo teve como objetivo identificar a prevalência de transtornos mentais em pacientes atendidos em ambulatório de psiquiatria infantil, a partir da análise do livro de registros de atendimento (anos de 2009 e 2010) preenchido por médicos residentes e docentes. Foram analisados dados de 109 pacientes, com idade entre 4 e 20 anos (média: II,08 $\pm 3,24$ ), sendo 85 do sexo masculino $(77,98 \%)$. Transtorno de déficit de atenção/hiperatividade $(n=59 ; 54,12 \%)$, retardo mental $(n=55 ; 50,45 \%)$ e transtorno de conduta $(n=34 ; 31,19 \%)$ foram os mais prevalentes. Para o sexo masculino, o mais prevalente foi transtorno de déficit de atenção e hiperatividade, e, para o feminino, retardo mental. As comorbidades mais prevalentes foram retardo mental, transtorno de conduta, transtorno desafiador e de oposição, episódio depressivo e transtorno da ansiedade. A maioria dos pacientes era do sexo masculino, com transtornos externalizantes e comorbidades. Os dados reafirmam a relevância de programas preventivos na atenção primária, na comunidade e nas escolas.
\end{abstract}

Palavras-chave: prevalência; transtornos mentais; transtornos do comportamento infantil; criança; adolescente.

\section{CHILDREN'S PSYCHIATRIC OUTPATIENT CLINIC: PREVALENCE OF MENTAL DISORDERS AMONG CHILDREN AND ADOLESCENTS}

\begin{abstract}
The objective of the study was to identify prevalence of mental disorders among patients served by a children's psychiatric outpatient unity based on the analysis of the register book of the unity (years: 2009 and 2010), filled by psychiatry residents and professors. Data from 109 patients, ages 4 to 20 (I I.08 \pm 3.24 ) was analyzed; 85 were male $(77.98 \%)$. Most prevalent disorders were attention-deficit/hyperactivity disorder $(n=59 ; 54.12 \%)$, mental retardation $(n=55 ; 50.45 \%)$ and conduct disorder $(n=34 ; 31.19 \%)$. For males the most prevalent disorder was attention deficit/hyperactivity and for females the most prevalent was mental retardation. The most prevalent comorbidities were mental retardation, conduct disorder, oppositional defiant disorder, depressive episode, and anxiety disorders. The majority of patients were male, had
\end{abstract}

1 Endereço para correspondência: Maria Cristina de Oliveira Santos Miyazaki, Faculdade de Medicina de São José do Rio Preto-SP, Avenida Brigadeiro Faria Lima, 5.416, Vila São Pedro - São José do Rio Preto - SP - Brasil. CEP: 15090-000. E-mail: cmiyazaki@famerp.br. 
Claudemir Marcos Machado, Andreia Mara Angelo Gonçalves Luiz, Altino Bessa Marques Filho, Maria Cristina de Oliveira Santos Miyazaki, Neide Aparecida Micelli Domingos, Eliana Márcia Sotello Cabrera

externalizing disorders and comorbidities. Data confirms the relevance of prevention programs in primary health care, community and schools.

Keywords: prevalence; mental disorders; child behavior disorders; child; adolescent.

AMBULATORIO DE PSIQUIATRÍA INFANTIL: PREVALENCIA DE LOS TRASTORNOS MENTALES EN NIÑOS Y ADOLESCENTES

\begin{abstract}
Resumen: Este estudio investigó la prevalencia de trastornos mentales entre pacientes atendidos en un ambulatorio de psiquiatría infantil mediante levantamiento de datos en el libro de registro de médicos residentes y profesores de psiquiatría (años 2009 y 2010). El estudio incluyó 109 pacientes con edad entre 4 y 20 años (media de II ,08 I $3,24), 85$ niños $(77,98 \%)$. Los trastornos más prevalentes fueron trastorno de déficit de atención con hiperactividad $(n=59 ; 54,12 \%)$, retardo mental $(n=55 ; 50,45 \%)$ y trastorno de conducta $(n=34 ; 31,19 \%)$. Para los niños el más prevaleciente fue el trastorno de déficit de atención con hiperactividad y para las niñas el retardo mental. Las comorbilidades más prevalecientes fueron retardo mental, trastorno de conducta, trastorno de oposición y desafio, depresión y ansiedad. La mayor parte de los pacientes fueron niños con trastornos externalizantes y otras comorbilidades. Los dados confirman la relevancia de los programas preventivos en la salud primaria, en la comunidad y en las escuelas.
\end{abstract}

Palabras clave: prevalencia; trastornos mentales; trastornos del comportamiento infantil; niño; adolescente.

Identificar a prevalência dos transtornos mentais na infância, bem como fatores de vulnerabilidade e de proteção, auxilia no delineamento de políticas de saúde, na distribuição de recursos, na prevenção e no tratamento de casos diagnosticados (Anselmi et al., 2008). Além de acarretarem prejuízos sobre o funcionamento global da criança, os transtornos mentais da infância tendem a persistir, frequentemente não recebem tratamento adequado e aumentam o risco para outros problemas, como abuso de substâncias, criminalidade, desemprego, mortalidade, dificuldades na educação dos filhos e transtornos mentais na vida adulta (Gale et al., 2012).

Existem dados indicando que de $50 \%$ a $75 \%$ dos transtornos mentais começam na infância e na adolescência (Kessler et al., 2007), embora estudos epidemiológicos ainda sejam escassos em países de baixa e média rendas (Petresco et al., 2014). Estima-se que nesses países, inclusive no Brasil, a prevalência de transtornos mentais na infância e adolescência varie entre $7 \%$ e $20 \%$, dependendo da região investigada, da exposição a fatores de risco e da metodologia empregada nos estudos (Kieling et al., 2011; Petresco et al., 2014).

O diagnóstico de um transtorno mental é habitualmente realizado a partir de critérios padronizados, como os da Classificação Internacional de Doenças (CID-10) ou do Manual diagnóstico e estatístico de transtornos mentais (DSM-IV). Os dois sistemas de classificação são convergentes e aceitos internacionalmente, e incluem, além dos critérios para diagnóstico de diferentes transtornos, o sofrimento e a incapacidade associados aos sintomas. Entretanto, críticas e insatisfação em relação ao seu uso são frequentes e incluem, por exemplo, seu difícil uso no mundo real, onde os pacientes apresentam múltiplos conjuntos de sintomas que se enquadram em 
diversos diagnósticos, falta de flexibilidade em relação à idade e ao sexo, e falta de sensibilidade quanto a questões culturais (Mash \& Hunsley, 2007).

Indicadores de fatores biológicos, psicológicos e sociais que influenciam o comportamento dos indivíduos e o desenvolvimento de transtornos mentais são consistentes e confirmam a relevância do modelo biopsicossocial de atenção à saúde: "A ocorrência concomitante de fatores de risco e de proteção complica a identificação das variáveis específicas, responsáveis pelo início e pela continuidade dos problemas de saúde mental" (Kieling et al., 2011, p. 1516). Fatores que aumentam o risco de transtornos mentais ao longo da vida incluem, por exemplo, aspectos genéticos, nutricionais, doenças, saúde mental dos pais ou responsáveis, ausência de lar estruturado, institucionalização, condições de extrema pobreza, maternidade/paternidade na adolescência, abuso de substâncias, desastres naturais, violência e negligência (Kieling et al., 2011; Anselmi et al., 2008). Por sua vez, crianças nascidas em famílias estruturadas, com pais instruídos, emocionalmente competentes, têm menor vulnerabilidade para transtornos mentais na infância (Kieling et al., 2011).

Familiares e professores têm importante papel na identificação dos transtornos mentais na infância (Vinocur \& Pereira, 2011). Geralmente, é responsabilidade dos pais reconhecer que o filho tem um problema e procurar o tratamento adequado. Entretanto, muitos não recorrem a ajuda profissional, acreditando que as alterações comportamentais são inerentes à idade e irão desaparecer com o desenvolvimento (Vinocur \& Pereira, 2011). Embora a maior parte dos transtornos mentais tenha início na infância e na adolescência, o tratamento efetivo só se inicia mais tarde, apesar de as evidências indicarem que intervenções precoces podem reduzir a gravidade do problema e prevenir o desenvolvimento de comorbidades (Girolamo, Dagani, Purcell, Cocchi, \& McGorry, 2012).

É preciso reconhecer que ainda existe preconceito social em relação aos tratamentos psicológicos e psiquiátricos, o que pode impedir muitos pais de procurar tratamento adequado para as suas crianças (Struch et al., 2008). Além disso, há a dificuldade de acesso a profissionais competentes, pouca disponibilidade de serviços de atenção à saúde mental nos postos de saúde e dificuldade de agendar consultas nos postos e instituições em que esses profissionais estão. Essas questões evidenciam a necessidade de aprimorar o planejamento administrativo do atendimento em saúde mental.

A capacitação de profissionais e de pais ou cuidadores permite, por meio de planejamento e de uma metodologia adequada, manejar transtornos mentais infantis de maneira satisfatória. $O$ delineamento de programas adequados de atendimento, entretanto, requer conhecer os transtornos mentais mais prevalentes em determinado serviço ou região. Visando responder a essa questão, foi elaborado o objetivo do presente estudo: identificar os principais transtornos mentais entre crianças e adolescentes atendidos no ambulatório de psiquiatria infantil do Hospital de Base. 
Claudemir Marcos Machado, Andreia Mara Angelo Gonçalves Luiz, Altino Bessa Marques Filho, Maria Cristina de Oliveira Santos Miyazaki, Neide Aparecida Micelli Domingos, Eliana Márcia Sotello Cabrera

\section{Método}

Este estudo tem caráter descritivo e foi aprovado pelo Comitê de Ética em Pesquisa da Faculdade de Medicina de São José do Rio Preto (Famerp).

Os dados foram obtidos por meio da análise das anotações do livro de registro do ambulatório de psiquiatria infantil do Hospital de Base de São José do Rio Preto/SP durante os anos de 2009 e 2010, período da duração do projeto de iniciação científica. As anotações do livro de registro foram feitas por médicos psiquiatras residentes e docentes.

\section{Resultados}

Dos 139 registros realizados no período, 109 estavam completos em relação ao diagnóstico e foram incluídos no estudo. A idade desses 109 pacientes variou de 4 a 20 anos (média 11,08 +3,24), sendo 85 do sexo masculino $(77,98 \%$ ) e 24 do sexo feminino $(22,02 \%)$. A Tabela 1 apresenta as taxas de prevalência dos diagnósticos realizados para ambos os sexos no período analisado.

Tabela I. Prevalência de transtornos mentais diagnosticados em ambulatório de psiquiatria infantil de um hospital de ensino (prevalência total e de acordo com o gênero)

\begin{tabular}{lllllll}
\hline & \multicolumn{2}{l}{ Total } & \multicolumn{2}{l}{ Masculino } & \multicolumn{2}{l}{ Feminino } \\
Diagnóstico & $\mathrm{n}=109$ & $\mathrm{n}=85$ & \multicolumn{2}{l}{$\mathrm{n}=24$} \\
\cline { 2 - 7 } & $\mathrm{n}$ & $\%$ & $\mathrm{n}$ & $\%$ & $\mathrm{n}$ & $\%$ \\
\hline Transtorno de déficit atenção e hiperatividade & 59 & 54,12 & 49 & 57,64 & 10 & 41,66 \\
Retardo mental & 55 & 50,45 & 37 & 43,52 & 18 & 75 \\
Transtorno de conduta & 34 & 31,19 & 26 & 30,58 & 8 & 33,33 \\
Episódio depressivo & 22 & 20,18 & 19 & 22,35 & 3 & 12,5 \\
Distúrbio desafiador e de oposição & 18 & 16,51 & 16 & 18,82 & 2 & 8,33 \\
Transtorno do pânico & 11 & 10,09 & 8 & 9,41 & 3 & 12,5 \\
Transtorno ansioso não especificado & 9 & 8,25 & 7 & 8,23 & 2 & 8,33 \\
Epilepsia & 7 & 6,42 & 7 & 8,23 & 0 & 0 \\
Transtorno global do desenvolvimento & 6 & 5,50 & 2 & 2,35 & 4 & 16,66 \\
Autismo & 6 & 5,50 & 3 & 3,52 & 3 & 12,5 \\
Transtorno obsessivo compulsivo & 6 & 5,50 & 6 & 7,05 & 0 & 0 \\
Transtorno específico: habilidades escolares & 5 & 4,58 & 3 & 3,52 & 2 & 8,33 \\
Síndrome comportamental associada a transtornos das & 4 & 3,66 & 4 & 4,70 & 0 & 0 \\
funções fisiológicas e a fatores físicos não especificados & & & & & & \\
Transtorno afetivo bipolar & 3 & 2,75 & 3 & 3,52 & 0 & 0 \\
Esquizofrenia & 2 & 1,83 & 2 & 2,35 & 0 & 0 \\
Transtorno personalidade com instabilidade emocional & 2 & 1,83 & 0 & 0 & 2 & 8,33 \\
\hline
\end{tabular}

Fonte: Elaborada pelos autores. 
Os pacientes foram também agrupados levando-se em conta a fase de desenvolvimento, segundo critérios da Organização Mundial de Saúde (OMS): infância (0-10 anos) e adolescência (11-20 anos). Os diagnósticos mais prevalentes entre crianças e adolescentes estão apresentados na Tabela 2.

Tabela 2. Diagnósticos mais prevalentes entre crianças e adolescentes atendidos em ambulatório de psiquiatria infantil de um hospital de ensino

\begin{tabular}{|c|c|c|}
\hline \multirow[t]{2}{*}{ Diagnóstico } & Crianças & Adolescentes \\
\hline & $\%$ & $\%$ \\
\hline Transtorno de déficit de atenção e hiperatividade & $1051 \%$ & $3^{\circ} 24,5 \%$ \\
\hline Retardo mental & $2 \circ 31,2 \%$ & $1 \circ 31,14 \%$ \\
\hline Transtorno de conduta & $3^{\circ} 14,87 \%$ & $2^{\circ} 24,61 \%$ \\
\hline Episódio depressivo & $4^{\circ} 8,5 \%$ & $4^{\circ} 11,47 \%$ \\
\hline Transtorno obsessivo compulsivo & $5^{\circ} 6,4 \%$ & $6^{\circ} 3,27 \%$ \\
\hline Outros transtornos da ansiedade & $6^{\circ} 4,25 \%$ & $4^{\circ} 11,47 \%$ \\
\hline Distúrbio desafiador e de oposição & $-0 \%$ & $4^{\circ} \mathrm{II}, 47 \%$ \\
\hline Transtorno global do desenvolvimento & $7^{\circ} 2,12 \%$ & $5^{\circ} 6,55 \%$ \\
\hline
\end{tabular}

Fonte: Elaborada pelos autores.

As comorbidades que apareceram com maior frequência para os diagnósticos mais prevalentes estão apresentadas na Tabela 3.

Tabela 3. Diagnósticos mais prevalentes e comorbidades entre crianças e adolescentes atendidos em ambulatório de psiquiatria

\begin{tabular}{|c|c|}
\hline Diagnóstico & Comorbidades mais frequentes \\
\hline $\left.1^{\circ}\right)$ Transtorno de déficit de atenção e & $1^{\circ}$ ) Retardo mental \\
\hline \multirow[t]{4}{*}{ hiperatividade } & $2^{\circ}$ ) Transtorno de conduta \\
\hline & $3^{\circ}$ ) Transtorno desafiador e de oposição \\
\hline & $\left.4^{\circ}\right)$ Episódio depressivo \\
\hline & $5^{\circ}$ ) Transtornos da ansiedade \\
\hline \multirow[t]{5}{*}{$\left.2^{\circ}\right)$ Retardo mental } & $\left.1^{\circ}\right)$ Transtorno de déficit de atenção e hiperatividade \\
\hline & $2^{\circ}$ ) Transtorno de conduta \\
\hline & $\left.3^{\circ}\right)$ Autismo \\
\hline & $4^{\circ}$ ) Episódio depressivo \\
\hline & $\left.5^{\circ}\right)$ Psicose \\
\hline \multirow[t]{5}{*}{$\left.3^{\circ}\right)$ Transtorno de conduta } & $\left.1^{\circ}\right)$ Transtorno de déficit de atenção e hiperatividade \\
\hline & $\left.2^{\circ}\right)$ Retardo mental \\
\hline & $3^{\circ}$ ) Transtorno desafiador e de oposição \\
\hline & $\left.4^{\circ}\right)$ Transtornos da ansiedade \\
\hline & $5^{\circ}$ ) Episódio depressivo \\
\hline
\end{tabular}

Fonte: Elaborada pelos autores. 


\section{Discussão}

Entre os registros avaliados no período do estudo, houve maior prevalência de atendimentos para o sexo masculino. Esse resultado é semelhante ao obtido em outros estudos brasileiros e internacionais com crianças encaminhadas para atendimento em saúde mental (Boaz, 2009; Petresco et al., 2014). Os dados sugerem que transtornos mentais são mais comuns em meninos do que em meninas e que os problemas aumentam no período próximo e durante a adolescência (Counselling Directory, 2014). Além disso, crianças com problemas externalizantes (como agressividade e transtorno de déficit de atenção e hiperatividade) têm maior probabilidade de ser encaminhadas para serviços de saúde mental que as com problemas internalizantes (como ansiedade e depressão). Como a prevalência de problemas externalizantes é maior entre os meninos, isso pode justificar o número superior de crianças do sexo masculino atendidas em serviços de saúde mental (Counselling Directory, 2014).

O transtorno de déficit de atenção e hiperatividade (TDAH), identificado como o mais prevalente neste estudo, é apontado como o diagnóstico realizado com maior frequência em serviços de saúde mental, responsável por altos custos em serviços de saúde do mundo todo. Trata-se de um problema crônico e que persiste, na maior parte dos casos, além da infância (American Academy of Pediatrics, 2011; Erskine et al., 2014).

O TDAH é mais prevalente para o sexo masculino (Smith, Barkley, \& Shapiro, 2007) embora autores sugiram que possa estar sendo subdiagnosticado no sexo feminino (Ramtekkar, Reiersen, Todorov, \& Todd, 2009). Considerado o problema "neurocomportamental mais comum da infância, pode afetar profundamente o rendimento acadêmico, o bem-estar e as interações sociais das crianças" (American Academy of Pediatrics, 2011).

$\mathrm{Na}$ avaliação do TDAH, outras condições devem ser investigadas e manejadas, uma vez que existem altas taxas de comorbidade com outros transtornos mentais, como ansiedade, depressão, transtorno de conduta, desafiador e de oposição, problemas de aprendizagem e tiques (American Academy of Pediatrics, 2011; Yüce, Zoroglu, Ceeylan, Kandemir, \& Karabekiroglu, 2013; Souza, Serra-Pinheiro, Fortes, \& Pinna, 2007; Hoza, Kaiser, \& Hurt, 2008; Barkley, 2006). Neste estudo, as principais comorbidades encontradas foram retardo mental, transtorno de conduta, transtorno desafiador e de oposição, episódio depressivo e transtornos da ansiedade.

A prevalência de TDAH entre crianças com retardo mental é alta, embora existam poucos estudos sobre o tema (Souza et al., 2007; Hässler \& Thome, 2012). Investigação sobre a comorbidade entre TDAH e síndrome de Down, por exemplo, identificou taxa positiva de 43,9\% entre as crianças avaliadas (Ekstein, Glick, Weill, Kay, \& Berger, 2011). Uma avaliação cuidadosa dessas crianças é necessária, portanto, para que o tratamento adequado possa ser indicado. Um exemplo da importância desse cuidado é fornecido por Hässler e Thome (2012): crianças com retardo mental podem ser mais suscetíveis aos efeitos colaterais do tratamento farmacológico utilizado habitualmente para o manejo do TDAH.

Existe alta taxa de comorbidade entre TDAH, transtorno desafiador e de oposição e transtorno de conduta (Yüce et al., 2013; Erskine et al., 2014). Sintomas de transtorno 
desafiador e de oposição estão presentes em parcela significativa de crianças com TDAH, e estas, por sua vez, têm grande chance de desenvolver transtorno de conduta (Patel, $\mathrm{Pa}$ tel, \& Patel, 2012). É importante notar que, embora o uso de farmacoterapia esteja estabelecido como um tratamento eficaz para o TDAH, a medicação não afeta diretamente problemas característicos das comorbidades, como o "comportamento opositivo/agressivo, os sintomas internalizantes, as habilidades sociais, o relacionamento entre pais e criança, e o funcionamento acadêmico" (Hoza et al., 2008, p. 197). Assim, existe grande interesse em intervenções psicossociais para o manejo do TDAH e de comorbidades, tanto isoladas como combinadas com medicação. Hoza et al. (2008) apresentam uma revisão sobre esses tratamentos, considerando especialmente aqueles baseados em evidências.

Existe hoje grande preocupação em avaliar custos dos serviços de saúde. Assim, é importante considerar que o transtorno de conduta tem custos diretos e indiretos superiores aos do TDAH (Merikangas, Nakamura, \& Kessler, 2009). Além do impacto do problema sobre a criança, família e contexto social, custos são um argumento adicional para incentivar pesquisas e intervenções baseadas em evidências para aqueles que apresentam TDAH e comorbidades.

Episódio depressivo e transtornos da ansiedade, comorbidades associadas ao TDAH, foram também identificados neste estudo. Em média, de $25 \%$ a $35 \%$ das crianças com TDAH apresentam transtornos da ansiedade (Barkley, 2006). Os sintomas de ansiedade nessas crianças são semelhantes aos apresentados por aquelas que têm apenas um transtorno da ansiedade. Sintomas de depressão também são comuns, e uma revisão da literatura identificou prevalência de transtorno depressivo maior ou transtorno distímico em $15 \%$ a $75 \%$ de crianças com TDAH. Assim, identificar e tratar ansiedade e depressão em crianças com TDAH é fundamental e tem um impacto positivo sobre a qualidade de vida (Barkley, 2006).

Como o transtorno mais prevalente identificado neste estudo foi o TDAH, é importante rever as diretrizes da American Academy of Pediatrics (2011) para a avaliação e o tratamento do transtorno: 1. avaliar em relação a TDAH pacientes de 4 a 18 anos com "problemas acadêmicos ou comportamentais e sintomas de déficit de atenção, hiperatividade ou impulsividade" (p. 6); 2. utilizar os critérios do Manual diagnóstico e estatístico de transtornos mentais, obter dados de múltiplos informantes (como pais e professores) e fazer diagnóstico diferencial em relação a outras condições; 3. avaliar comorbidades frequentes no TDAH; 4. considerar e tratar TDAH como um problema crônico de saúde, que requer cuidados especiais; 5 a. encaminhar pré-escolares (4-5 anos) para tratamento baseado em evidências; $5 \mathrm{~b}$. para escolares (6-11 anos), prescrever medicação aprovada pela Food and Drug Administration (FDA) para TDAH e/ou tratamento baseado em evidências, incluindo o contexto escolar no plano terapêutico; $5 c$. para adolescentes (12-18 anos), prescrever medicação e terapia comportamental, de preferência ambas.

Quando os diagnósticos são divididos por faixa etária, os meninos apresentaram como mais prevalente TDAH, seguido por retardo mental e transtorno de conduta. As meninas, por sua vez, apresentaram como mais prevalente retardo mental, seguido por TDAH 
e transtorno de conduta. As altas taxas de comorbidade identificadas neste estudo indicam a necessidade da atenção dos profissionais ao realizarem um diagnóstico: identificar também as comorbidades que devem ser alvo de atenção.

A intervenção precoce do TDAH pode reduzir a gravidade e a persistência do problema, bem como prevenir comorbidades (Girolamo et al., 2012). Assim, profissionais precisam estar atentos para o possível diagnóstico deste e de outros transtornos mentais prevalentes na infância e adolescência, uma vez que têm um importante papel na identificação e no manejo desses problemas. Existem evidências indicando que a intervenção durante os estágios iniciais dos transtornos mentais da infância e adolescência "pode auxiliar a reduzir a gravidade e/ou persistência do transtorno inicialmente diagnosticado e prevenir transtornos secundários" (Girolamo et al., 2012, p. 47).

O estudo realizado permitiu concluir que a maior prevalência de atendimento foi para o sexo masculino e que crianças com problemas externalizantes foram encaminhadas com maior frequência. Os dados indicam a necessidade de programas preventivos na comunidade e nas escolas, bem como a importância de alertar pais e professores em relação à identificação precoce de sintomas de transtornos mentais, inclusive dos internalizantes.

Outro importante aspecto a ser considerado em relação aos dados deste estudo refere-se ao fato de os diagnósticos das crianças e adolescentes terem sido levantados do livro de registros do ambulatório, preenchido pelos médicos psiquiatras. A impossibilidade de utilizar vários registros, por estarem incompletos, indica a necessidade de treinamento dos profissionais quanto à importância de documentar o atendimento aos pacientes. Embora a instituição disponha hoje de um sistema informatizado, que dispensa o livro de registros, é preciso treinar os profissionais para o uso adequado do sistema. Registros de atendimento em saúde auxiliam a proporcionar melhor qualidade de cuidados. Permitem ainda identificar a prevalência dos diferentes transtornos mentais, para que programas adequados de atendimento possam ser disponibilizados, de acordo com as necessidades da população.

Mais estudos sobre prevalência de transtornos mentais entre crianças e adolescentes ainda são necessários para identificar necessidades regionais específicas. Esses estudos devem incluir diferentes recursos da comunidade onde a população recebe assistência na área de saúde mental e estudos populacionais.

\section{Referências}

American Academy of Pediatrics (2011). ADHD: clinical practice guideline for the diagnosis, evaluation, and treatment of attention-deficit/hyperactivity disorder in children and adolescents. Pediatrics, 128. Recuperado em 10 janeiro, 2014, de http://pediatrics.aappublications.org/content/early/2011/10/14/peds.2011-2654.

Anselmi, L., Barros, F. C., Minten, G. C., Gigante, D. P., Horta, B. L., \& Victora, C. G. (2008). Prevalência e determinantes precoces dos transtornos mentais comuns na coorte de nascimentos de 1982, Pelotas, RS. Revista de Saúde Pública, 42(supl. 2), 26-33. 
Barkley, R.A. (2006). Transtornos co-mórbidos, adaptação social e familiar e subtipos. In R. A. Barkely. Transtorno de déficit de atenção/hiperatividade. Manual para diagnóstico e tratamento (3a ed., pp.196-230). Porto Alegre: Artmed.

Boaz, C. (2009). Caracterização das queixas apresentadas por meninos e meninas encaminhados a clínicas-escola. Dissertação de mestrado, Pontifícia Universidade Católica do Rio Grande do Sul, Porto Alegre, RS, Brasil. Recuperado em 22 fevereiro, 2014, de http://repositorio.pucrs.br/dspace/handle/10923/4869.

Counselling Directory. (2014). Key statistics about children and young people. Recuperado em 14 março, 2014, de http://www.counseling-directory.org.uk/childrenstats.html.

Ekstein, S., Glick, B., Weill, M., Kay, B., \& Berger, I. (2011). Down syndrome and attention-deficit/hyperactivity disorder (ADHD). Journal of Child Neurology, 26, 1290-1295.

Erskine, H. E., Ferrari, A. J., Polanczyk, G. V., Moffitt, T. E., Vos, T., Whiteford, H. A., \& Scott, J. G. (2014). The global burden of conduct disorder and attention-deficit/ hyperactivity disorder in 2010. Journal of Child Psychology and Psychiatry, 22. Recuperado em 10 março, 2014, de http://onlinelibrary.wiley.com/doi/10.1111/ jcpp.12186/full.

Gale, C. R., Batty, G. D., Osborn, D. P., Tynelius P., Whitley, E., \& Rasmussen, F. (2012). Association of mental disorders in early adult life and later hospital psychiatric admission and mortality in a cohort study of more than 1 million men. Archives of General Psychiatry, 69, 823-831.

Girolamo, G., Dagani, J., Purcell, R., Cocchi, A., \& McGorry P. D. (2012). Age of onset of mental disorders and use of mental health services: needs, opportunities and obstacles. Epidemiology and Psychiatric Sciences, 21(1), 47-57.

Hässler, F., \& Thome, J. (2012). Mental retardation and ADHD. Zeitschrift für Kinder- und Jugendpsychiatrie und Psychotherapie, 40(12), 83-93.

Hoza, B., Kaiser, N., \& Hurt, E. (2008). Evidence-based treatments for attention-deficit/hyperactive disorder (ADHD). In R. G. Steele, T. D. Elkin \& M. C. Roberts (Eds.). Handbook of evidence-based therapies for children and adolescents. Bridging science and practice (pp. 197-219). New York: Springer.

Kessler, R. C., Amminger, G. P., Aguilar-Gaxiola, S., Alonso, J., Lee, S., \& Ustun, T. B. (2007). Age of onset of mental disorders: a review of recent literature. Current Opinion in Psychiatry, 20, 359-364.

Kieling, C., Baker-Henningham, H., Belfer, M., Conti, G., Ertem, I., Omigbodun, O., Rohde, L. A., Srinath, S., Ulkuer, N., \& Rahman, A. (2011). Child and adolescent mental health worldwide: evidence for action. The Lancet, 378, 1515-1525. 
Mash, E. J., \& Hunsley, J. (2007). Assessment of child and family disturbance: a developmental-systems approach. In E. J. Mash \& R. A. Barkley (Eds.). Assessment of childhood disorders (pp. 3-52). New York: Guilford.

Merikangas, K. R., Nakamura, E. F., \& Kessler, R. C. (2009). Epidemiology of mental disorders in children and adolescents, State of the art. Dialogues in Clinical Neuroscience, 11, 7-20.

Patel, N., Patel, M., \& Patel, H. (2012). ADHD and comorbid conditions. In J. M. Norvillis (Ed.). Current directions in ADHD and its treatment. Recuperado em 10 março, 2014, de http://www.intechopen.com/books/current-directions-in-adhd-and-its-treatment/adhd-and-comorbidity.

Petresco, S., Anselmi, L., Santos, I. S., Barros, A. J. D., Fleitlich-Bilyk, B., Barros, F. C., \& Matijasevich, A. (2014). Prevalence and comorbidity of psychiatric disorders among 6-year-old children: 2004 Pelotas Birth Cohort. Social Psychiatry and Psychiatric Epidemiology. Recuperado em 10 março, 2014, de http://download. springer.com/static/pdf/651/art\%253A10.1007\%252Fs00127-014-0826-z. pdf?auth66=1395947677_d34e4c08ab6c8183f24e50cc5deb3477\&ext=.pdf.

Ramtekkar, U. P., Reiersen, A. M., Todorov, A. A., \& Todd, R. D. (2009). Sex and age differences in attention-deficit/hyperactivity disorder symptoms and diagnoses: implications for DSM-V and ICD-11. Child \& Adolescent Psychiatry, 49(3), 217-228.

Smith, B. H., Barkley, R. A., \& Shapiro, C. J. (2007). Attention-deficit/hyperactivity disorder. In E. J. Mash \& R. A. Barkely (Eds.). Assessment of childhood disorders (4th ed., pp. 53-123). New York: Guilford.

Souza, I. G. S., Serra-Pinheiro, M. A., Fortes, D., \& Pinna, C. (2007). Dificuldades no diagnóstico de TDAH em crianças. Jornal Brasileiro de Psiquiatria, 56(supl. 1), 14-18.

Struch, N., Levav, I., Shereshevsky, Y., Baidani-Auerbach, A., Lachman, M., Daniel, N., \& Zehavi, T. (2008). Stigma experienced by persons under psychiatric care. The Israel Journal of Psychiatry and Related Sciences, 45(3), 210-218.

Vinocur, E., \& Pereira, H. V. F. S. (2011). Avaliação dos transtornos de comportamento na infância. Revista Hospital Universitário Pedro Ernesto, 10, 26-34.

Yüce, M., Zoroglu, S. S., Ceeylan, M. F., Kandemir, H., \& Karabekiroglu, K. (2013). Psychiatric comorbidity distribution and diversities in children and adolescents with attention-deficit/hyperactivity disorder: a study from Turkey. Neuropsychiatric Disease and Treatment, 9, 1791-1799.

Submissão: 20.06.2013

Aceitação: 08.05.2014 